\title{
Wireless Multisensory Interaction in an Intelligent Rehabilitation Environment
}

\author{
Miguel Oliver, José Pascual Molina, Francisco Montero, Pascual González, and \\ Antonio Fernández-Caballero \\ 1 Instituto de Investigación en Informática de Albacete (I3A), 02071-Albacete, Spain \\ 2 Universidad de Castilla-La Mancha, Departamento de Sistemas Informáticos, \\ 02071-Albacete, Spain \\ oliveredsi.uclm.es
}

\begin{abstract}
Today, the population is aging, and this is becoming a problem for current health systems, as each day it has to invest more money in treating the elderly. Rehabilitation of elderly patients with physical disabilities is one of these problems that everyday incur greater overhead to health care. This paper provides a gerontechnology-based solution by proposing a multisensory system for rehabilitation in an intelligent environment. The proposal enables helping needed people and thus reducing the cost of health care.
\end{abstract}

Keywords: Gerontechnology, Rehabilitation, Intelligent environments, Multisensory interaction, Wireless sensor networks

\section{Introduction}

Nowadays, the population is aging, and this is becoming a problem for current health systems, as each day it has to invest more money in treating the elderly [1], [2]. Rehabilitation is one of these problems that everyday incur greater overhead to health care.

Gerontechnology is an interdisciplinary field of scientific research in which technology is directed towards the aspirations and opportunities for the older persons [3]. Gerontechnology aims at good health, full social participation and independent living up to a high age, be it research, development or design of products and services to increase the quality of life. The development of gerontechnology-based support systems for rehabilitation follows two distinct paths. On one hand, some approaches make use of different specialized hardware to aid the elderly patient in his/her recovery [4]. Generally, these solutions often require a large outlay of money, making access to these products not universal. On the other hand, some other systems propose the use of depth sensors like Kinect to develop a rehabilitation system [5]. This makes the system affordable to the general public, but the interaction is not completely satisfactory in some cases.

In this paper we propose to combine the advantages of both types of systems discussed above. For this, a Kinect sensor is used to pick up the movements made by the elderly in his/her rehabilitation process. In addition, the Wiimote actuator is used to 
provide haptic sensations to the patient. Thus, the resulting communication is enriched and the elderly is informed about the complete rehabilitation process.

In the next section some rehabilitation systems will be shown, these will help us to create a system of rehabilitation for elderly people that will be displayed in the third section. Finally the conclusions obtained during the development of the rehabilitation system are discussed, which lead us to say that the project developed allows the rehabilitation of elderly people from home, which means saving time and money for both the patient and rehabilitation center.

\section{Some Current Rehabilitation Systems}

In the literature there are several examples of the use of depth sensors such as the Kinect sensor as part of support systems in rehabilitation [6]. In a recent paper [5] the possibility of using the Kinect sensor for the rehabilitation of patients with motor impairments is studied. The system is used by two young participants, resulting in improved patient motivation in rehabilitation and improving stretching exercise performance. Another paper [7] contains a study which tests the Kinect sensor as a substitute for classic assisted rehabilitation, offering the user the possibility of rehabilitation at home. The result of the study shows that the reliability in gesture recognition is between $88.0 \%$ and $92.2 \%$. Moreover, the study proves that the participants find more fun and ease to use in the Kinect than in the conventional system. Another development and evaluation of a rehabilitation system based on Kinect has been presented [8]. The proposed system is composed of two modules. The first consists of a set of support stretching exercises for physical rehabilitation, and the second, in a data analyzer which detects posture and wrong actions on the user. Also, a comparison of low cost sensor Kinect and sensor motion capture OptiTrac V100:R2 of the company NaturalPoint has been presented [9]. The comparison raises that the Kinect sensor provides an acceptable performance that is competitive with the sensor OptiTrac V100:R2, but with a much lower price. The proposal allows universal accessibility to computerized rehabilitation treatments. In addition, other papers (e.g. [10], [11], [12]) propose the use of devices such as the Kinect sensor and the development of games to help patients in their rehabilitation.

Moreover, to date, some physical systems have been implemented for the rehabilitation of patients. VirtualRehab [13], developed by company Virtualware Group, is a product for the rehabilitation of patients with any degree of physical disability. The main feature of its software is that it deals with the issue of rehabilitation as a game. This ensures the software to have a playful component, which induces the user to feel more comfortable during the treatment. At present, the system has 9 games/exercises that the physiotherapist assigns to a patient depending on his/her disability.

Teki [14] is a project of the Basque Health Service (Osakidetza). The project seeks to improve the quality of life of chronic patients, providing a tool to monitor clinical status and to facilitate greater communication between the patient and the specialist, thereby improving the care of the user at home by using new information technologies.Teki allows the collection of clinical data using medical devices, the response to symptom questionnaires and the recording of self-administration of medication, which allows to remotely monitor the patient. 
Toyra [15] is a rehabilitation product developed by the company Indra. It is aimed to the rehabilitation of the upper body, and for it, makes use of Kinect sensor and a set of sensors that have to be attached to the body. These sensors measure the movement of the human body and transmit it to the computer, which adds this data to the information collected by the Kinect, to form a set of more accurate data. The system is divided into Assisted Toyra, which is aimed for use in specialized centers with the supervision of a physiotherapist, and Independent Toyra, with is aimed at the rehabilitation of the patient at home.

Brontes Processing [16] is a company dedicated to developing games which base their interaction on webcams for personal computers. At present, the company offers two products for use in rehabilitation: SeeMe and Home rehabilitation. The SeeMe system is used for the rehabilitation of patients in specialized centers, requiring the support of specialized doctors for proper operation. Now, Home rehabilitation unlike the previous one, is used for the rehabilitation of patients at home.

Reflexion [17] is a system developed by the medical West Health Institute. Its main purpose is to maintain the interest of the patient throughout the treatment and to inform the therapist about the correction in the conduct of the stretching exercise. The specialist selects and adapts the rehabilitation exercises to each patient, who performs the exercises at home or at a place suitable for use by a computer and a Kinect sensor.

Lastly, KineLabs [18] is a project at the Hong Kong Polytechnic University for help in the rehabilitation of the elderly and people with physical disabilities. The system currently contains three stretching exercises which are aimed at coordination of the upper limbs, coordination of the lower limbs and trunk balance training.

There are two disadvantages in the systems proposed in this section. The first disadvantage is that these systems are based on predetermined exercises which are assigned to the patients, this means that the exercises are generic and cannot adjust to the peculiarities of each of these patients. The second disadvantage of these systems is the use of visual and auditory stimuli, for patients with visual and audible deficiencies, this can make, that the rehabilitation occurs incorrectly. Considering these disadvantages, we will create the system of rehabilitation for elderly patients.

\section{A New Proposal of a Rehabilitation System}

Our system is being developed to assist in the rehabilitation of elderly patients with physical disabilities. To accomplish this, the therapist establishes a set of stretching exercises that the patients have to do. The system is responsible of handling the rehabilitation exercises and evaluating their adequacy, taking as example the stretching exercises provided by the monitor. In addition, the system provides feedback to the user in relation to the correctness of the exercise performed. During the system development, we considered the option of providing an audible feedback to the user, but this stimulus can be confusing and annoying in the moment that there are several rehabilitation patients in a same room, as it usually happens in rehabilitation groups. Also the elderly people, the common user of this system, may have problems with this stimulus due to their hearing problems. Because of these reasons, we decided to add an audio feedback to the system, but augmented with another sensory channel. Therefore, we performed a 


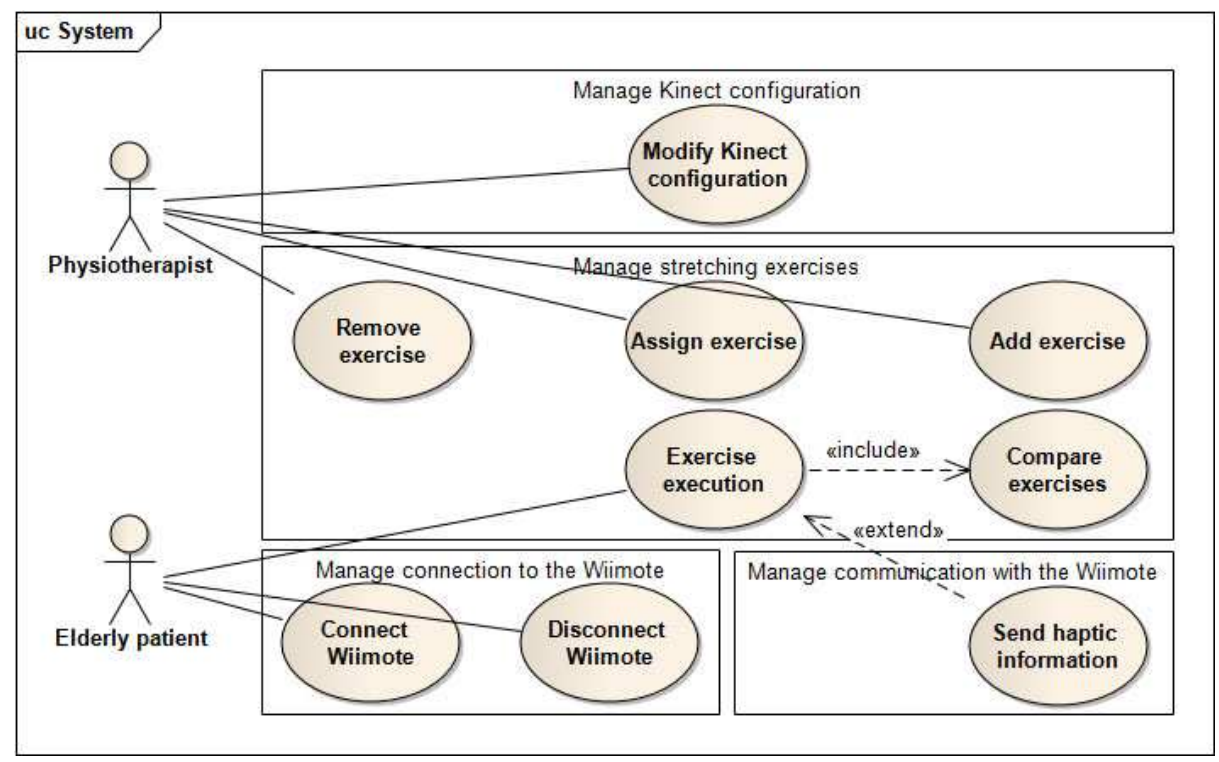

Fig. 1. Use case diagram of the rehabilitation system

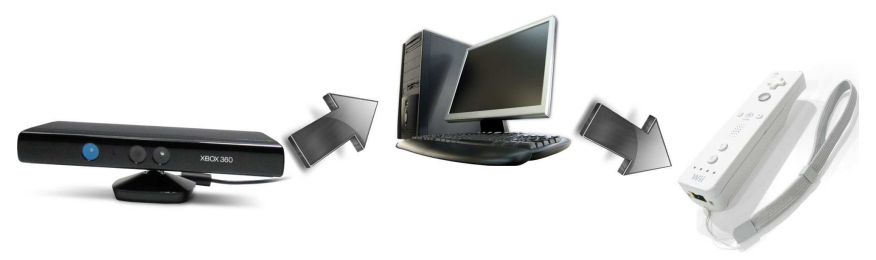

Fig. 2. Diagram of hardware components of the system

study on haptics to supplement the auditory canal. As a result, we reached the idea of using vibration motors to enhance the communication between the user and the system. More specifically, we chose to use a Wiimote game controller used on the Wii console.

Fig. 1 shows a use case diagram of the developed system. It describes how the system is composed of two users, the therapist and the elderly patient, as well as the tasks performed by each of them. The most important tasks are described later on.

Fig. 2 shows the hardware devices that compose the rehabilitation system. The heart of the system is the computer. It is responsible for processing the signals of all devices and for running the developed software. The Kinect device is connected to the PC via USB and is responsible for collecting and preprocessing the images of the therapist and the elderly patient. The Wiimote device is connected to the PC using a Bluetooth connection and is responsible for providing haptic feedback to the patient. This way the elderly patient determines if he/she is performing the stretching exercise correctly. The arrows in the figure indicate the flow of information. 


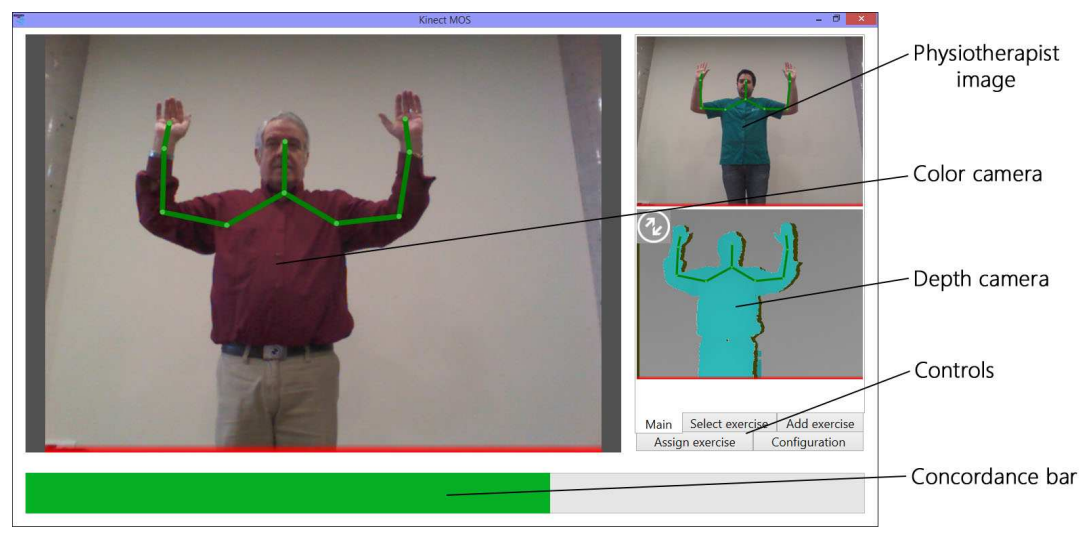

Fig. 3. Elderly person performing a shoulder rehabilitation exercise

In first place, the developed system looks for a Wiimote device to provide haptic feedback to the user. If there is at least one paired device, using Bluetooth, the system recognizes it and connect to it. If there is no Wiimote connected to the computer, the rehabilitation system warns about this fact and asks the user if he/she wants to search again a device or to run without haptic feedback. Fig. 3 shows the main interface of the program and an elderly patient performing an stretching exercise proposed by a physiotherapist. The center-left of the screen shows the image captured in real-time by the Kinect sensor. On the right side of the interface there is a tab control that offers four different tabs used both by the elderly patient and the physical therapist to navigate the application and to interact with it.

Next, the operation of the system is described according to the functionality required by each system role.

\subsection{Physiotherapist}

The role of the physiotherapist in the system is relatively simple. He/she adds the stretching exercises, assigns these rehabilitation exercises to elderly patients, and adjusts the characteristics of the system to adapt it to the environment where the Kinect device is located.

1. Add exercise. To add new stretching exercises to the system, the therapist must select "Add exercise". Once done, a similar interface to that shown in Fig. 4 appears. The therapist assigns a name to the rehabilitation exercise and he/she adopts a position with his/her body that fits the desired rehabilitation. For this part, he/she makes use of the image displayed in the main window which collects the image in real-time of the therapist as well as his/her skeleton. Once the correct posture for rehabilitation is adopted and the Kinect sensor has recognized it, he/she adds the position to the system database.

2. Assign exercise. To assign stretching exercises to each patient, the therapist must select "Assign exercise". This will show all the rehabilitation exercises stored in 


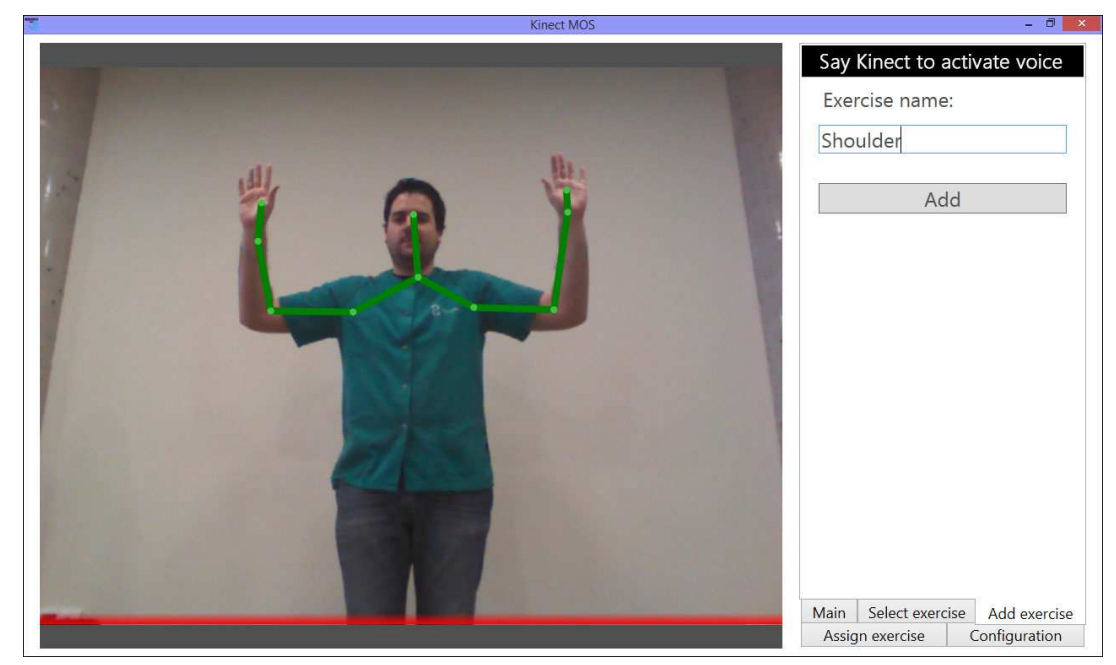

Fig. 4. Physiotherapist creating a shoulder rehabilitation exercise

system and provides the option to enable or disable this exercise for a particular user. So the elderly patient can choose only those exercises that the specialty was assigned him/her.

3. Modify Kinect configuration. Another task is to establish the correct parameters of the Kinect sensor to make it work in a proper way. This is achieved by selecting the "Configuration". The configuration options allow to adjust many of the properties of the sensor. Some of the most important features are: the resolution of image capture, color and depth; the recognition of the user standing (Normal mode) or sitting (Near mode); the determination of the recognized user in case there are more users captured by the camera; the inclination of sensor. This allows the Kinect to perfectly conform to the medium in which it is located, thereby improving the resulting interaction with the system.

\subsection{Elderly Patient}

The role of the elderly patient in the system is to perform stretching exercises that the physiotherapist has previously established. To do this, he/she just selects one of the available exercises and does it as well as possible.

Exercise execution enables the user to select one of the stretching exercises assigned by the physiotherapist. The interface shows all available positions which are composed of a descriptive text and an image showing the therapist performing the rehabilitation exercise. The user just double clicks on the desired exercise to start. Once the exercise has been selected, an interface similar to that of Fig. 3 is automatically displayed. The elderly patient aided by the real-time image captured by the sensor should adopts a similar posture to the sample. To ensure that the patient's posture is similar to the physiotherapist's one, the bottom bar shows the degree of concordance between the two 
positions. This encourages the users to perform better exercises and this helps in their rehabilitation. Fig. 3 shows a patient performing an stretching exercise, the Wiimote controller, which can be held by hand or stored in his pocket, vibrate when the elderly person performing the rehabilitation exercise correctly. The vibration frequency and intensity depends on the correction in the development of exercise, becoming maximal when the exercise is completely well performed. So, an additional help identifies how he/she is conducting an exercise, helping him/her to continue the rehabilitation.

\section{Discussion and Conclusions}

Most systems proposed in the literature make use of rehabilitation games. The main objective is to involve the user in the process of rehabilitation and to prevent the treatment abandonment. Our system follows this principle to make more pleasant the elderly patient rehabilitation. In contrast, the previously proposed systems make use of default stretching exercises, which are assigned to the patient in accordance with the necessary rehabilitation. This can make that the specific requirements of a elderly patient are not covered. Our system improves this deficiency by ensuring that the stretching exercises are specifically tailored to the elderly patient and fully meet his/her needs. Visual and audio interaction are used in these systems. But this also causes a problem in rehabilitation of patients with audible and visual impairment, which also coincides with the common user who uses to require rehabilitation, the elderly people. Our system has added haptic interaction to help elderly patients in identifying how they are performing the rehabilitation exercises.

The distinguishing characteristics of our system allow the rehabilitation of a general public and their ease of use allows access to people with no computer skills. Also, they reduce the rehabilitation cost of both the elderly patient and the rehabilitation center, as they enable the rehabilitation of elderly patients at their own home, thereby saving the constant supervision necessary in common rehabilitation. At this moment, the systems allows the execution of rehabilitation exercises which do not require high precision, e.g. arms and legs. However we are working in the improvement of the system to perform precision exercises, such as finger rehabilitation.

\section{Acknowledgements}

This work was partially supported by Spanish Ministerio de Economía y Competitividad / FEDER under TIN2010-20845-C03-01 and TIN2012-34003 grants. We would also like to thank RedAUTI (512RT0461) for the comments and suggestions which allowed to greatly improve the quality of the paper.

\section{References}

1. Costa, A., Castillo, J.C., Novais, P., Fernández-Caballero, A., Simoes, R.: Sensor-driven agenda for intelligent home care of the elderly. Expert Systems with Applications 39:15, 12192-12204. 2012. 
2. Castillo, J.A., Carneiro, D., Serrano-Cuerda, J., Novais, P., Fernández-Caballero, A., Neves, J.: A multi-modal approach for activity classification and fall detection. International Journal of Systems Science 45:4, 810-824. 2014.

3. Harrington, T.L., Harrington, M.K.: Gerontechnology: Why and How. Shaker Publishing BV. 2000

4. Schönauer, C., Pintaric, T., Kaufmann, H., Jansen-Kosterink, S., Vollenbroek-Hutten, M.: Chronic pain rehabilitation with a serious game using multimodal input. Proceedings of the 2011 International Conference on Virtual Rehabilitation, 1-8. 2011.

5. Chang, Y., Chen, S., Huang, J.: A Kinect-based system for physical rehabilitation: A pilot study for young adults with motor disabilities. Research in Developmental Disabilities 32:6, 2566-2570. 2011.

6. Fuentes, J.A., Oliver, M., Montero, F., Fernández-Caballero, A., Fernández, M.A.: Towards usability evaluation of multimodal assistive technologies using RGB-D sensors. Lecture Notes in Computer Science 7931, 210-219. 2013.

7. Davaasambuu, E., Chiang, C., Chiang, J., Chen, Y., Bilgee, S.: A Microsoft Kinect based virtual rehabilitation system. Proceedings of the 5th International Conference on Frontiers of Information Technology, Applications and Tools, 44-50. 2012.

8. Freitas, D., Da Gama, A., Figueiredo, L., Chaves, T., Marques-Oliveira, D., Teichrieb, V., Araújo, C.: Development and evaluation of a Kinect based motor rehabilitation game. Proceedings of Simposio Brasileiro de Jogos e Entretenimento Digital, 144-153. 2012.

9. Chang, C., Lange, B., Zhang, M., Koenig, S., Requejo, P., Somboon, N., Sawchuk, A., Rizzo, A.: Towards pervasive physical rehabilitation using Microsoft Kinect. Proceedings of the 6th International Conference on Pervasive Computing Technologies for Healthcare, 1-4. 2012.

10. Lange, B., Chang, C., Suma, E., Newman, B., Rizzo, A., Bolas, M.: Development and evaluation of low cost game-based balance rehabilitation tool using the Microsoft Kinect sensor. Proceedings of the 33rd Annual International Conference of the IEEE Engineering in Medicine and Biology Society, 1831-1834. 2011.

11. Pirovano, M., Mainetti, R., Baud-Bovy, G., Lanzi, P., Borghese, N.: Self-adaptive games for rehabilitation at home. Proceedings of the IEEE Conference on Computational Intelligence and Games, 179-186. 2012.

12. Da Gama, A., Chaves, T., Figueiredo, L., Teichrieb, V.: Improving motor rehabilitation process through a natural interaction based system using Kinect sensor. Proceedings of the 2012 IEEE Symposium on 3D User Interfaces, 145-146. 2012.

13. VirtualRehab. http://virtualrehab.info. 2013.

14. Teki. http://www.osakidetza.euskadi.net. 2013.

15. Toyra. http://www.toyra.org. 2013.

16. Brontes Processing. http://www.brontesprocessing.com. 2013.

17. Reflexion. http://www.westhealth.org/institute/our-priorities/reflexion. 2013.

18. KineLabs. www.polyu.edu.hk/kinelabs. 2013. 